\title{
Symptom overlap between schizophrenia and bipolar mood disorder: Diagnostic issues
}

\author{
Vivek Bambole $^{1}$, Megan Johnston ${ }^{2}$, Nilesh Shah ${ }^{1}$, Sushma Sonavane ${ }^{3}$, \\ Avinash Desouza $^{3}$, Amresh Shrivastava ${ }^{4,5 *}$ \\ ${ }^{1}$ Department of Psychiatry, Lokmanya Tilak Municipal Medical College, University of Mumbai, Mumbai, India \\ ${ }^{2}$ Department of Psychology, University of Toronto, Toronto, Canada \\ ${ }^{3}$ Department of Psychiatry, Lokmanya Tilak Municipal Medical College and General Hospital, Mumbai, India \\ ${ }^{4}$ Mental Health Resource Foundation (PRERANA Charitable Trust) and Silver Mind Hospital, Mumbai, India \\ ${ }^{5}$ Department of Psychiatry, The University of Western Ontario, Lawson Health Research Institute, London, Canada; \\ Email: *Amresh.edu@gmail.com
}

Received 18 April 2013; revised 16 May 2013; accepted 24 May 2013

Copyright (C) 2013 Vivek Bambole et al. This is an open access article distributed under the Creative Commons Attribution License, which permits unrestricted use, distribution, and reproduction in any medium, provided the original work is properly cited.

\begin{abstract}
Although the Kraepelinian classification paradigm is widely used, observations of overlapping boundaries among the symptoms associated with bipolar disorder and schizophrenia are beginning to challenge this dichotomy. The objective of this research was to explore the symptoms of individuals diagnosed with schizophrenia and with bipolar mood disorder in order to determine the frequency of symptom overlap. One hundred patients of a psychiatry ward were divided into two main groups based on their diagnosis-schizophrenia or bipolar mood disorder. Chi-square analyses were used to determine whether the symptoms measured in this study differed between individuals diagnosed with schizophrenia and those diagnosed with bipolar mood disorder. The results suggest that both positive/manic symptoms and negative/depressive symptoms are present in individuals diagnosed with schizophrenia and with bipolar mood disorder and, consequently, they do not present a reliable means of differentiating between these two groups. These findings have many implications for the ways in which mental illness is conceptualized and classified. Treatment efforts and interventions may be enhanced if a more dimensional approach to diagnosing mental illness is utilized.
\end{abstract}

Keywords: Classification; Depressive Symptoms; Mania; Diagnosis

\section{INTRODUCTION}

"Corresponding author.
As initially defined by Kraepelin, current classification systems assume two major psychotic disorders: dementia praecox and manic-depressive [1]. Although this paradigm is widely used, observations of overlapping boundaries among the symptoms associated with bipolar disorder and schizophrenia are beginning to challenge this dichotomy. Since Kraepelin's original proposal, the concept of schizophrenia has evolved, exposing the flaws in applying a dichotomous classification system. In particular, overlapping features of both schizophrenia and bipolar disorder, such as psychotic symptoms, do not allow for definitive differential diagnosis. Further, these disorders are now recognized as syndromes but not a singular disease entity.

Kendler and colleagues determined six underlying disease syndromes based on a latent class analysis to patients who had been treated for psychotic and affective disorders [2]. These researchers then validated the syndromes on the basis of independent demographic, familial, clinical, and course data. This proposition of six types of illness suggests that the spectrum of psychotic and affective disorders is much more complex than Kraepelin believed. Further evidence that schizophrenia and bipolar mood disorder may be two points on a continued spectrum of the same disease rather than separate clinical entities comes from considerable overlap in phenomenology and neurobiology $[3,4]$. Some patients also show a change in diagnosis from schizophrenia to bipolar mood disorder or vice versa over time [5]. Additionally, patients of schizophrenia sometimes show inter episodic recovery as seen in bipolar mood disorder [6].

Previous research has demonstrated that symptom overlap does occur in these disorders. For example, Cardno et al. described the lifetime rate of occurrence of 
manic syndrome as $9 \%$ in schizophrenic patients [7]. Additionally, various studies have reported comorbid depressive symptoms or syndromes in patients diagnosed with schizophrenia, with the prevalence of these symptoms varying widely between $7 \%$ and $83 \%$ of patients with schizophrenia [8-10]. Psychotic symptoms have also been found to accompany affective disorders to a certain degree. In fact, psychotic major depression has been proposed as an independent diagnosis classification based on evidence that it differs from major depressive disorder $[11,12]$.

Importantly, the term "schizoaffective disorder" was introduced to explain the overlapping symptoms of psychotic disorders and mood disorders, first defined by Kasanin [13]. A diagnosis of schizoaffective disorder is frequently used to describe a psychotic person with significant symptoms of depression and/or mania, but there is continued debate regarding the clinical validity and utility of this diagnosis [14]. The relation between schizophrenia and the major mood disorders remains ambiguous, with imprecise and variable boundaries between the two. Schizoaffective disorder has been at the center of the continuum between schizophrenia and major mood disorders, and beliefs about its nature vary considerably.

The objective of this research was to explore the symptoms of individuals diagnosed with schizophrenia and with bipolar mood disorder in order to determine the frequency of symptom overlap. Psychometric scales typically used to assess clinical symptoms of schizophrenia and bipolar mood disorder were used to measure the symptoms of both disorders in all patients participating in the study.

\section{METHODS}

This study was undertaken by the Department of Psychiatry in a tertiary municipal teaching hospital with attached medical college. The patients were recruited from the psychiatry ward. The sample consisted of 100 consecutive patients suffering from schizophrenia and bipolar mood disorder admitted in the psychiatry ward from December 2007 to July 2009 with a minimum duration of illness of two years. Inclusion criteria for the study were as follows: patient and/or relative were willing to participate in the study, the age of 18 to 65 years, and patient was suffering from schizophrenia or bipolar mood disorder according to DSM IV -TR with a minimum duration of illness of two years. Patients were excluded from the study if they lacked objective data or they were diagnosed with any other psychiatric disorder.

\subsection{Sample}

The 100 participants (47 female) in the present research had a mean age of $35.86(\mathrm{SD}=12.36$; range $=18-65)$.
Twenty-two participants had completed college, 64 had primary level education, and the remaining 14 were illiterate. The majority of participants were married $(\mathrm{N}=60)$, whereas 34 participants were unmarried, and six were divorced or separated. Almost all participants were of low or middle socio-economic status (lower $=63$; middle $=$ 33 ; upper $=4$ ).

Out of the 100 patients, 72 had schizophrenia and 28 had bipolar mood disorder. Out of 72 patients of schizophrenia, 58 (80.56\%) patients were paranoid, 11 (15.28\%) were residual, $2(2.78 \%)$ were disorganized and $1(1.39 \%)$ was catatonic type. Out of 28 patients of bipolar mood disorder, $23(82.14 \%)$ had a manic episode, $3(10.71 \%)$ had mixed episodes, and $2(7.14 \%)$ had a depressive episode. Fifteen patients had co-morbid substance dependence, of which 11 had alcohol dependence, three had cannabis dependence and one had benzodiazepine dependence. Thirty patients had a family history of psychiatric illness, the large majority of which was schizophrenia $(\mathrm{n}=28)$.

\subsection{Measures}

\subsubsection{Positive Symptoms}

Positive symptoms were assessed in the present study with the Scale for Assessment of Positive Symptoms (SAPS) [15]. This scale is designed to assess the positive symptoms that primarily occur in schizophrenia, including hallucinations, delusions, bizarre behavior, and positive formal thought disorder. Items are rated on a scale from 0 (none) to 5 (severe). The scale is composed of 30 items assessing specific symptoms and five items of global ratings. For scores of $0=$ absent and $1=$ questionable, the symptom was considered as absent; for the scores of 2 - 5 (mild to severe) it was considered as present. The measure of symptoms was thus a binomial present vs. absent variable.

\subsubsection{Negative Symptoms}

Negative symptoms were assessed using the Scale for Assessment of Negative Symptoms (SANS) [16]. This scale is designed to assess the negative symptoms primarily occurring in schizophrenia, including affective flattening or blunting, alogia (slowed thought processes), avolition/apathy, anhedonia/asociality, and difficulties with attention. Items are rated on a scale from 0 (none) to 5 (severe). A present $(2-5)$ vs. absent $(0-1)$ binomial variable was used in the analyses. The scale is composed of 20 items assessing specific symptoms and five global ratings.

\subsubsection{Depressive Symptoms}

The Hamilton Depression Rating Scale (HDRS) was used to measure depressive symptoms in the present sample [17]. This is a 17 item scale that focuses on the 
melancholic and physical symptoms of depression. Most items are rated on a scale of 0 (absent) to 4 (high level of symptom), although some items are rated on a three or four point scale. A present $(2-4)$ vs. absent $(0$ - 1) binomial variable was used in the analyses. Symptoms assessed include insomnia, anxiety, depressed mood, and weight loss.

\subsubsection{Manic Symptoms}

Symptoms of mania were assessed with the Young Mania Rating Scale (YMRS) [18]. This scale is composed of 11 items designed to assess the severity of symptoms of a manic episode. Items are rated on a scale from 0 (absent) to 4 (high level of symptom) and include elevated mood, increased motor activity, irritability, and disruptive/aggressive behavior. A present (2 - 4) vs. absent $(0-1)$ binomial variable was used in the analyses.

\subsection{Procedure}

Participants were recruited from the psychiatry ward of a municipal teaching hospital with attached medical college. The patients and/or relatives were explained the nature of the study. An informed consent was taken from the patient and/or relatives. Patients were then interviewed using the semi structured pro-forma designed for this research and relevant data was collected for the individual case. Symptoms typically associated with schizophrenia were assessed with the SAPS and the SANS. Symptoms typically associated with bipolar mood disorder were assessed with HDRS and the YMRS. The data was then pooled, tabulated and subjected to statistical analysis. Patients were divided into two main groups based on their diagnosis - schizophrenia or bipolar mood disorder - and the data were analyzed according to these two main groups. Chi-square analyses were used to determine whether the symptoms measured in this study differed between individuals diagnosed with schizophrenia and those diagnosed with bipolar mood disorder.

\section{RESULTS}

\subsection{Sample Characteristics}

Initial analyses were undertaken to explore whether participants with schizophrenia $(\mathrm{N}=72)$ differed from participants with bipolar mood disorder $(\mathrm{N}=28)$ on the demographic variables measured in this study. An independent samples $t$-test demonstrated no differences in the age of individuals in these two groups, $t(98)=0.18, p=$ 0.86 . Chi-square analyses were used to examine the remaining variables. No differences were found in level of education $\left(\chi^{2}(2)=3.00, p=0.22\right)$, socio-economic status $\left(\chi^{2}(2)=1.21, p=0.55\right)$, presence of substance abuse $\left(\chi^{2}\right.$ $(1)=1.26, p=0.26)$, or family history of mental illness $\left(\chi^{2}(1)=1.36, p=0.24\right)$. Analyses that approached sig- nificance were gender differences $\left(\chi^{2}(1)=3.45, p=0.06\right)$ and differences in marital status $\left(\chi^{2}(2)=5.60, p=0.06\right)$. There were a larger proportion of individuals with bipolar mood disorder who were male and who were married.

\subsection{Positive Symptoms of Schizophrenia}

Chi-square analyses were used to compare patients diagnosed with schizophrenia to those diagnosed with bipolar mood disorder on SAPS ratings (see Table 1). Global ratings of severity of hallucinations differed between the groups, $\chi^{2}(1)=10.70, p=0.001$. Severe hallucinations were more prevalent in the schizophrenia group $(50.00 \%)$ than in the bipolar mood disorder group $(14.29 \%)$. In terms of the six specific symptoms of hallucinations, only auditory hallucinations differed between the two groups, $\chi^{2}(1)=8.61, p=0.003$, with a higher prevalence found in the schizophrenia group $(45.83 \%$ vs. $14.29 \%)$. Global ratings of delusions did not differ between the two groups, $\chi^{2}(1)=0.54, p=0.46$. However, the two groups differed significantly on four of the 12 specific delusion symptoms. Individuals in the schizophrenia group had higher ratings of persecutory delusions $(84.72 \%$ vs. $64.29 \%)$ and delusions of reference $(73.61 \%$ vs. $17.86 \%)$, whereas the bipolar mood disorder group had higher ratings of grandiose delusions $(78.57 \%$ vs. $23.61 \%)$ and religious delusions ( $28.57 \%$ vs. $4.17 \%)$.

The schizophrenia group and the bipolar mood disorder group did not differ in global ratings of bizarre behavior, $\chi^{2}(1)=0.002, p=0.89$, but they did differ on one specific symptom of bizarre behavior-social/sexual behavior, $\chi^{2}(1)=4.19, p=0.04$. The bipolar mood disorder group evidenced more of this behavior $(57.14 \%$ vs. $34.72 \%$ ). The two groups differed significantly in the frequency of severe symptoms of positive formal thought disorder, $\chi^{2}(1)=35.70, p<0.001$. Individuals in the bipolar mood disorder group were more likely to have severe symptoms than individuals in the schizophrenia group ( $85.71 \%$ vs. $20.83 \%$, respectively). Six of the eight symptoms of positive formal thought disorder were more prevalent in the bipolar mood disorder group (see Table 1).

\subsection{Negative Symptoms of Schizophrenia}

Chi-square analyses were also used to compare the schizophrenia group to the bipolar mood disorder group on SANS ratings (see Table 2). Global severity ratings of affective flattening/blunting differed between the two groups, $\chi^{2}(1)=45.60, p<0.001$. Severe ratings were more prevalent in the schizophrenia group than in the bipolar mood disorder group $(83.33 \%$ vs. $10.71 \%)$. Five of the seven symptoms associated with affective flattening/blunting were more frequent in the schizophrenia group (see Table 2). Global ratings of alogia also dif- 
Table 1. Scale for assessment of positive symptoms (SAPS) ratings.

\begin{tabular}{|c|c|c|c|}
\hline Symptoms & $\operatorname{SCZ}(N=72)$ & BMD $(\mathrm{N}=28)$ & Chi-square and $p$-value \\
\hline Hallucination $(\mathrm{N}=40)$ & $36(50.00 \%)$ & $4(14.29 \%)$ & $\chi^{2}(1)=10.70, p=0.001$ \\
\hline Auditory hallucination $(\mathrm{N}=37)$ & $33(45.83 \%)$ & $4(14.29 \%)$ & $\chi^{2}(1)=8.61, p=0.003$ \\
\hline Voices commenting $(\mathrm{N}=3)$ & $3(4.17 \%)$ & $0(0.00 \%)$ & $\chi^{2}(1)=1.20, p=0.27$ \\
\hline Voices conversing $(\mathrm{N}=14)$ & $12(16.67 \%)$ & $2(7.14 \%)$ & $\chi^{2}(1)=1.52, p=0.22$ \\
\hline Somatic/tactile hallucination $(\mathrm{N}=1)$ & $1(1.39 \%)$ & $0(0.00 \%)$ & $\chi^{2}(1)=0.39, p=0.53$ \\
\hline Olfactory hallucination $(\mathrm{N}=0)$ & $0(0.00 \%)$ & $0(0.00 \%)$ & \\
\hline Visual hallucination $(\mathrm{N}=04)$ & $4(5.56 \%)$ & $0(0.00 \%)$ & $\chi^{2}(1)=1.62, p=0.20$ \\
\hline Delusion $(\mathrm{N}=83)$ & $61(84.72 \%)$ & $22(78.57 \%)$ & $\chi^{2}(1)=0.54, p=0.46$ \\
\hline Persecutory delusion $(\mathrm{N}=79)$ & $61(84.72 \%)$ & $18(64.29 \%)$ & $\chi^{2}(1)=5.08, p=0.02$ \\
\hline Delusion of jealousy $(\mathrm{N}=25)$ & $18(25.00 \%)$ & $7(25.00 \%)$ & $\chi^{2}(1)=0.00, p=1.00$ \\
\hline Delusion of guilt/sin $(\mathrm{N}=4)$ & $2(2.78 \%)$ & $2(7.14 \%)$ & $\chi^{2}(1)=1.00, p=0.32$ \\
\hline Grandiose delusion $(\mathrm{N}=41)$ & $17(23.61 \%)$ & $22(78.57 \%)$ & $\chi^{2}(1)=24.60, p<0.001$ \\
\hline Religious delusion $(\mathrm{N}=11)$ & $3(4.17 \%)$ & $8(28.57 \%)$ & $\chi^{2}(1)=12.30, p<0.001$ \\
\hline Somatic delusion $(\mathrm{N}=6)$ & $5(6.94 \%)$ & $1(3.57 \%)$ & $\chi^{2}(1)=0.41, p=0.52$ \\
\hline Delusion: reference $(\mathrm{N}=58)$ & $53(73.61 \%)$ & $5(17.86 \%)$ & $\chi^{2}(1)=25.70, p<0.001$ \\
\hline Delusion: being controlled $(\mathrm{N}=7)$ & $7(9.72 \%)$ & $0(0.00 \%)$ & $\chi^{2}(1)=2.93, p=0.09$ \\
\hline Delusion: mind reading $(\mathrm{N}=2)$ & $2(2.78 \%)$ & $0(0.00 \%)$ & $\chi^{2}(1)=0.79, p=0.37$ \\
\hline Thought broadcasting $(\mathrm{N}=4)$ & $4(5.56 \%)$ & $0(0.00 \%)$ & $\chi^{2}(1)=1.62, p=0.20$ \\
\hline Thought insertion $(\mathrm{N}=2)$ & $2(2.78 \%)$ & $0(0.00 \%)$ & $\chi^{2}(1)=0.79, p=0.37$ \\
\hline Thought withdrawal $(\mathrm{N}=0)$ & $0(0.00 \%)$ & $0(0.00 \%)$ & \\
\hline Bizarre behavior $(\mathrm{N}=74)$ & $53(73.61 \%)$ & $21(75.00 \%)$ & $\chi^{2}(1)=0.002, p=0.89$ \\
\hline Clothing/appearance $(\mathrm{N}=40)$ & $32(44.44 \%)$ & $8(28.57 \%)$ & $\chi^{2}(1)=2.12, p=0.15$ \\
\hline Social/sexual behavior $(\mathrm{N}=41)$ & $25(34.72 \%)$ & $16(57.14 \%)$ & $\chi^{2}(1)=4.19, p=0.04$ \\
\hline Aggression and agitated behavior $(\mathrm{N}=71)$ & $51(70.83 \%)$ & $20(71.43 \%)$ & $\chi^{2}(1)=0.003, p=0.95$ \\
\hline Repetitive and stereotyped behavior $(\mathrm{N}=1)$ & $1(1.39 \%)$ & $0(0.00 \%)$ & $\chi^{2}(1)=0.39, p=0.53$ \\
\hline Positive formal thought disorder $^{*}(\mathrm{~N}=35)$ & $15(20.83 \%)$ & $24(85.71 \%)$ & $\chi^{2}(1)=35.70, p<0.001$ \\
\hline Derailment $(\mathrm{N}=6)$ & $2(2.78 \%)$ & $4(14.29 \%)$ & $\chi^{2}(1)=4.73, p=0.03$ \\
\hline Tangentiality $(\mathrm{N}=11)$ & $4(5.56 \%)$ & $7(25.00 \%)$ & $\chi^{2}(1)=7.79, p=0.01$ \\
\hline Incoherence $(\mathrm{N}=6)$ & $4(5.56 \%)$ & $2(7.14 \%)$ & $\chi^{2}(1)=0.09, p=0.76$ \\
\hline Illogicality $(\mathrm{N}=11)$ & $8(11.11 \%)$ & $3(10.71 \%)$ & $\chi^{2}(1)=0.003, p=0.96$ \\
\hline Circumstantiality $(\mathrm{N}=23)$ & $6(8.33 \%)$ & $17(60.71 \%)$ & $\chi^{2}(1)=31.20, p<0.001$ \\
\hline Pressure of speech $(\mathrm{N}=39)$ & $15(20.83 \%)$ & $24(85.71 \%)$ & $\chi^{2}(1)=35.70, p<0.001$ \\
\hline Distractible speech $(\mathrm{N}=17)$ & $7(9.72 \%)$ & $10(35.71 \%)$ & $\chi^{2}(1)=9.65, p=0.002$ \\
\hline Clanging $(\mathrm{N}=12)$ & $1(1.39 \%)$ & $11(39.29 \%)$ & $\chi^{2}(1)=27.40, p<0.001$ \\
\hline
\end{tabular}

*Symptoms of positive formal thought disorder include derailment, tangentiality, incoherence, illogicality, circumstantiality, pressure of speech, distractible speech and clanging.

fered between the two groups, $\chi^{2}(1)=26.00, p<0.001$, with severe ratings more frequent in those diagnosed with schizophrenia $(63.89 \%$ vs. $7.14 \%$ of those diagnosed with bipolar mood disorder). Three of the four symptoms of alogia were more prevalent in the schizophrenia group (see Table 2). Severe ratings of avolitionapathy were also more prevalent in schizophrenia than in bipolar mood disorder, $\chi^{2}(1)=15.70, p<0.001(93.06 \%$ vs. $60.71 \%$, respectively) and all three symptoms were also more prevalent in the schizophrenia group (see Table 2). Similarly, severe ratings of anhedonia-asociality, and all four of its associated symptoms, were more frequent in the schizophrenia group, $\chi^{2}(1)=42.60, p<$
0.001 (90.28\% vs. $25.00 \%$ in the bipolar mood disorder group). Finally, global ratings of severe attention problems differed between the groups, $\chi^{2}(1)=4.26, p=0.04$ (83.33\% in schizophrenia vs. $64.29 \%$ in bipolar mood disorder). One of two symptoms of attentional difficulties was more prevalent in the schizophrenia groupsocial inactiveness, $\chi^{2}(1)=36.60, p<0.001(81.94 \%$ in schizophrenia vs. $14.29 \%$ in bipolar mood disorder).

\subsection{Manic Symptoms of Bipolar Mood Disorder}

Eleven symptoms of mania assessed with the YMRS were compared between the schizophrenia group and the 
Table 2. Scale for assessment of negative symptoms (SANS) ratings.

\begin{tabular}{|c|c|c|c|}
\hline Symptoms & $\operatorname{SCZ}(\mathrm{N}=72)$ & BMD $(\mathrm{N}=28)$ & Chi-square and $p$-value \\
\hline Affective flattening/blunting $(\mathrm{N}=63)$ & $60(83.33 \%)$ & $3(10.71 \%)$ & $\chi^{2}(1)=45.60, p<0.001$ \\
\hline Unchanged facial expression $(\mathrm{N}=46)$ & $43(59.72 \%)$ & $3(10.71 \%)$ & $\chi^{2}(1)=19.50, p<0.001$ \\
\hline Decreased spontaneous movements $(\mathrm{N}=42)$ & $41(56.94 \%)$ & $1(3.57 \%)$ & $\chi^{2}(1)=0.79, p=0.37$ \\
\hline Paucity of expressive gesture $(\mathrm{N}=54)$ & $51(70.83 \%)$ & $3(10.71 \%)$ & $\chi^{2}(1)=23.60, p<0.001$ \\
\hline Poor eye contact $(\mathrm{N}=50)$ & $47(65.28 \%)$ & $3(10.71 \%)$ & $\chi^{2}(1)=24.00, p<0.001$ \\
\hline Affective non responsibility $(\mathrm{N}=56)$ & $53(73.61 \%)$ & $3(10.71 \%)$ & $\chi^{2}(1)=32.40, p<0.001$ \\
\hline Lack of vocal inflection $(\mathrm{N}=50)$ & $48(66.67 \%)$ & $2(7.14 \%)$ & $\chi^{2}(1)=28.60, p<0.001$ \\
\hline Inappropriate affect $(\mathrm{N}=12)$ & $10(13.89 \%)$ & $2(7.14 \%)$ & $\chi^{2}(1)=0.87, p=0.35$ \\
\hline Alogia $(\mathrm{N}=47)$ & $46(63.89 \%)$ & $2(7.14 \%)$ & $\chi^{2}(1)=26.00, p<0.001$ \\
\hline Poverty of speech $(\mathrm{N}=46)$ & $44(61.11 \%)$ & $2(7.14 \%)$ & $\chi^{2}(1)=23.60, p<0.001$ \\
\hline Poverty of content $(\mathrm{N}=46)$ & $44(61.11 \%)$ & $2(7.14 \%)$ & $\chi^{2}(1)=23.60, p<0.001$ \\
\hline Blocking $(\mathrm{N}=0)$ & $0(0.00 \%)$ & $0(0.00 \%)$ & \\
\hline Increased latency $(\mathrm{N}=42)$ & $41(56.94 \%)$ & $1(3.57 \%)$ & $\chi^{2}(1)=23.60, p<0.001$ \\
\hline Avolition-apathy $^{*}(\mathrm{~N}=84)$ & $67(93.06 \%)$ & $17(60.71 \%)$ & $\chi^{2}(1)=15.70, p<0.001$ \\
\hline Grooming/hygiene $(\mathrm{N}=40)$ & $34(47.22 \%)$ & $6(21.43 \%)$ & $\chi^{2}(1)=5.59, p=0.02$ \\
\hline Impersistence at work $(\mathrm{N}=84)$ & $67(93.06 \%)$ & $17(60.71 \%)$ & $\chi^{2}(1)=15.70, p<0.001$ \\
\hline Physical anergia $(\mathrm{N}=60)$ & $57(79.17 \%)$ & $3(10.71 \%)$ & $\chi^{2}(1)=39.40, p<0.001$ \\
\hline Anhedonia-asociality $(\mathrm{N}=72)$ & $65(90.28 \%)$ & $7(25.00 \%)$ & $\chi^{2}(1)=42.60, p<0.001$ \\
\hline Recreational interest and activity $(\mathrm{N}=72)$ & $63(87.50 \%)$ & $9(32.14 \%)$ & $\chi^{2}(1)=30.60, p<0.001$ \\
\hline Sexual activity $(\mathrm{N}=21)$ & $21(29.17 \%)$ & $0(0.00 \%)$ & $\chi^{2}(1)=10.30, p=0.001$ \\
\hline Ability to feel intimacy and closeness $(\mathrm{N}=65)$ & $61(84.72 \%)$ & $4(14.29 \%)$ & $\chi^{2}(1)=44.00, p<0.001$ \\
\hline Relationship $(\mathrm{N}=67)$ & $61(84.72 \%)$ & $6(21.43 \%)$ & $\chi^{2}(1)=36.50, p<0.001$ \\
\hline Attention $(\mathrm{N}=78)$ & $60(83.33 \%)$ & $18(64.29 \%)$ & $\chi^{2}(1)=4.26, p=0.04$ \\
\hline Social inactiveness $(\mathrm{N}=63)$ & $59(81.94 \%)$ & $4(14.29 \%)$ & $\chi^{2}(1)=36.60, p<0.001$ \\
\hline Inactive during MSE $(\mathrm{N}=75)$ & $57(79.17 \%)$ & $18(64.29 \%)$ & $\chi^{2}(1)=2.38, p=0.12$ \\
\hline
\end{tabular}

*Symptoms of avoliation - apathy include grooming and hygiene, impersistence at work and physical anergia, due to presence of impersistence at work patients of bipolar mood disorder had high score.

bipolar mood disorder group using chi-square analyses (see Table 3). Individuals in the bipolar mood disorder group were significantly more likely to demonstrate five of these symptoms: elated $\operatorname{mood}\left(\chi^{2}(1)=45.30, p\right.$ $<0.001 ; 92.86 \%$ vs. $19.44 \%)$, increased motor activity $\left(\chi^{2}(1)=45.30, p<0.001 ; 92.86 \%\right.$ vs. $\left.19.44 \%\right)$, sexual interest $\left(\chi^{2}(1)=19.60, p<0.001 ; 57.14 \%\right.$ vs. $13.89 \%)$, speech $\left(\chi^{2}(1)=22.60, p<0.001 ; 75.00 \%\right.$ vs. $23.61 \%)$, and language and thought disorder $\left(\chi^{2}(1)=\right.$ $35.70, p<0.001 ; 85.71 \%$ vs. $20.83 \%$ ). The remaining symptoms of mania did not differ between the two groups (see Table 3 ).

\subsection{Depressive Symptoms of Bipolar Mood Disorder}

Seventeen depressive symptoms assessed with the HDRS were compared between the schizophrenia group and the bipolar mood disorder group using chi-square analyses (see Table 4). Only four of these symptoms differed between the two groups, and in all cases individuals in the schizophrenia group were more likely to demonstrate the symptoms than individuals in the bipolar mood disorder group. Specifically, the schizophrenia group had higher levels of insomnia (early: $\chi^{2}(1)=27.30, p<0.001$, $65.28 \%$ vs. $7.14 \%$; middle: $\chi^{2}(1)=16.60, p<0.001$, $51.39 \%$ vs. $7.14 \%$; late: $\chi^{2}(1)=7.78, p=0.005,34.72 \%$ vs. $7.14 \%)$, work and activities $\left(\chi^{2}(1)=15.70, p<0.001\right.$; $93.06 \%$ vs. $60.71 \%)$, retardation $\left(\chi^{2}(1)=9.40, p=0.002\right.$; $43.06 \%$ vs. $10.71 \%)$, and paranoid symptoms $\left(\chi^{2}(1)=\right.$ $5.08, p=0.02 ; 84.72 \%$ vs. $64.29 \%$ ).

\subsection{Frequency of Overlap in Symptoms}

In order to determine the number of patients with overlapping symptoms of schizophrenia and bipolar mood disorder, cut-off scores were used to classify those patients who showed a significant level of symptoms on each measure. The cut-off score used to determine a manic episode on the YMRS was greater than or equal to 20 out of 60 (Young et al., 1978). A depressive episode on the HDRS was considered to be scores greater than or equal to 11 out of 53 [19]. A minimal combined score of 42 on both the SAPS and the SANS, which was present 
Table 3. Young mania rating scale (YMRS) ratings.

\begin{tabular}{|c|c|c|c|}
\hline Symptoms & $\operatorname{SCZ}(\mathrm{N}=72)$ & BMD $(\mathrm{N}=28)$ & Chi-square and $p$-value \\
\hline Elated $\operatorname{mood}(\mathrm{N}=40)$ & $14(19.44 \%)$ & $26(92.86 \%)$ & $\chi^{2}(1)=45.30, p<0.001$ \\
\hline Increased motor activity $(\mathrm{N}=40)$ & $14(19.44 \%)$ & $26(92.86 \%)$ & $\chi^{2}(1)=45.30, p<0.001$ \\
\hline Sexual interest $(N=26)$ & $10(13.89 \%)$ & $16(57.14 \%)$ & $\chi^{2}(1)=19.60, p<0.001$ \\
\hline Sleep $(N=91)$ & $65(90.28 \%)$ & $26(92.86 \%)$ & $\chi^{2}(1)=0.16, p=0.69$ \\
\hline Irritability $(\mathrm{N}=88)$ & $65(90.28 \%)$ & $23(82.14 \%)$ & $\chi^{2}(1)=1.26, p=0.26$ \\
\hline Speech $(N=39)$ & $17(23.61 \%)$ & $21(75.00 \%)$ & $\chi^{2}(1)=22.60, p<0.001$ \\
\hline Language and thought disorder $(\mathrm{N}=39)$ & $15(20.83 \%)$ & $24(85.71 \%)$ & $\chi^{2}(1)=35.70, p<0.001$ \\
\hline Content $(\mathrm{N}=83)$ & $61(84.72 \%)$ & $22(78.57 \%)$ & $\chi^{2}(1)=0.54, p=0.46$ \\
\hline Disruptive-aggressive behavior $(\mathrm{N}=71)$ & $51(70.83 \%)$ & $20(71.43 \%)$ & $\chi^{2}(1)=0.003, p=0.95$ \\
\hline Appearance $(\mathrm{N}=40)$ & $32(44.44 \%)$ & $8(28.57 \%)$ & $\chi^{2}(1)=2.12, p=0.15$ \\
\hline Insight $(\mathrm{N}=90)$ & $67(93.06 \%)$ & $23(82.14 \%)$ & $\chi^{2}(1)=2.67, p=0.10$ \\
\hline
\end{tabular}

Table 4. Hamilton depression rating scale (HDRS) ratings.

\begin{tabular}{|c|c|c|c|}
\hline Symptoms & $\operatorname{SCZ}(N=72)$ & BMD (N = 28) & Chi-square and $p$-value \\
\hline Depressed mood $(\mathrm{N}=19)$ & $13(18.06 \%)$ & $6(21.43 \%)$ & $\chi^{2}(1)=0.15, p=0.70$ \\
\hline Feeling of guilt $(\mathrm{N}=5)$ & $2(2.78 \%)$ & $3(10.71 \%)$ & $\chi^{2}(1)=2.67, p=0.10$ \\
\hline Suicide $(\mathrm{N}=8)$ & $4(5.56 \%)$ & $4(14.29 \%)$ & $\chi^{2}(1)=2.09, p=0.15$ \\
\hline Insomnia-early $(\mathrm{N}=49)$ & $47(65.28 \%)$ & $2(7.14 \%)$ & $\chi^{2}(1)=27.30, p<0.001$ \\
\hline Insomnia-middle $(\mathrm{N}=39)$ & $37(51.39 \%)$ & $2(7.14 \%)$ & $\chi^{2}(1)=16.60, p<0.001$ \\
\hline Insomnia-late $(N=27)$ & $25(34.72 \%)$ & $2(7.14 \%)$ & $\chi^{2}(1)=7.78, p=0.005$ \\
\hline Work and activities $(\mathrm{N}=84)$ & $67(93.06 \%)$ & $17(60.71 \%)$ & $\chi^{2}(1)=15.70, p<0.001$ \\
\hline Retardation $(\mathrm{N}=34)$ & $31(43.06 \%)$ & $3(10.71 \%)$ & $\chi^{2}(1)=9.40, p=0.002$ \\
\hline Agitation $(\mathrm{N}=71)$ & $51(70.83 \%)$ & $20(71.43 \%)$ & $\chi^{2}(1)=0.003, p=0.95$ \\
\hline Anxiety-psychic $(\mathrm{N}=18)$ & $15(20.83 \%)$ & $3(10.71 \%)$ & $\chi^{2}(1)=1.40, p=0.24$ \\
\hline Anxiety-somatic $(\mathrm{N}=8)$ & $6(8.33 \%)$ & $2(7.14 \%)$ & $\chi^{2}(1)=0.04, p=0.84$ \\
\hline \multicolumn{4}{|l|}{ Somatic symptoms } \\
\hline Somatic symptoms-gastrointestinal $(\mathrm{N}=8)$ & $6(8.33 \%)$ & $2(7.14 \%)$ & $\chi^{2}(1)=0.04, p=0.84$ \\
\hline Somatic symptoms-general $(\mathrm{N}=12)$ & $8(11.11 \%)$ & $4(14.29 \%)$ & $\chi^{2}(1)=0.19, p=0.66$ \\
\hline Genital symptoms $(\mathrm{N}=6)$ & $5(6.94 \%)$ & $1(3.57 \%)$ & $\chi^{2}(1)=0.41, p=0.52$ \\
\hline Hypochondriosis $(\mathrm{N}=6)$ & $4(5.56 \%)$ & $2(7.14 \%)$ & $\chi^{2}(1)=0.09, p=0.76$ \\
\hline Loss of weight $(\mathrm{N}=16)$ & $14(19.44 \%)$ & $2(7.14 \%)$ & $\chi^{2}(1)=2.27, p=0.13$ \\
\hline Insight $(\mathrm{N}=90)$ & $67(93.06 \%)$ & $23(82.14 \%)$ & $\chi^{2}(1)=2.67, p=0.10$ \\
\hline Diurnal variation $(\mathrm{N}=1)$ & $0(0.00 \%)$ & $1(3.57 \%)$ & $\chi^{2}(1)=2.60, p=0.11$ \\
\hline Depersonalization/derealization $(\mathrm{N}=1)$ & $1(1.39 \%)$ & $0(0.00 \%)$ & $\chi^{2}(1)=0.39, p=0.53$ \\
\hline Paranoid symptoms $(\mathrm{N}=79)$ & $61(84.72 \%)$ & $18(64.29 \%)$ & $\chi^{2}(1)=5.08, p=0.02$ \\
\hline Obsessive and compulsive symptoms $(\mathrm{N}=3)$ & $3(4.17 \%)$ & $0(0.00 \%)$ & $\chi^{2}(1)=1.20, p=0.27$ \\
\hline
\end{tabular}

in clinically diagnosed cases of schizophrenia, was taken as a cut-off score for schizophrenia symptoms in patients of bipolar mood disorder.

Out of 72 patients diagnosed with schizophrenia, 47 (65.28\%) patients showed overlapping symptoms of mania and depression that were significant enough scores on psychometric rating scales (YMRS and HDRS) to allow for separate diagnoses of a manic episode or major depressive disorder. Out of these 47 patients, 21 patients
(29.17\%) had combined symptoms of both mania and depression, 15 patients $(20.83 \%)$ showed only symptoms of mania and 11 patients $(15.28 \%$ ) showed only symptoms of depression (see Table 5). Thus, the total numbers of patients diagnosed with schizophrenia demonstrating clinically significant manic features was $36(50.00 \%)$ and depressive feature was $32(44.44 \%)$.

Out of 28 patients diagnosed with bipolar mood disorder, 17 patients $(60.71 \%)$ showed overlapping symp- 
Table 5. Frequency of symptom overlap.

\begin{tabular}{lc}
\hline \multicolumn{1}{c}{ Schizophrenia } & No. of patients (72) \\
\hline Overlap with both mania and depression & $21(29.17 \%)$ \\
Overlap with mania only & $15(20.83 \%)$ \\
Overlap with depression only & $11(15.28 \%)$ \\
No overlap & $25(34.72 \%)$ \\
\hline \multicolumn{1}{c}{ Bipolar Mood Disorder } & No. of patients (28) \\
\hline Overlap with schizophrenia & $17(60.71 \%)$ \\
No overlap & $11(39.29 \%)$ \\
\hline
\end{tabular}

toms of schizophrenia that were clinically significant on psychometric rating scales (SAPS and SANS). Thus, 11 patients with bipolar mood disorder (39.29\%) did not show significant levels of overlapping symptoms.

\section{DISCUSSION}

The present research explored positive and negative symptoms typically associated with schizophrenia and depressive and manic symptoms associated with bipolar mood disorder in patients diagnosed with either of these illnesses. The results suggest significant overlap in symptoms between the two disorders. When examining the positive and negative symptoms used to diagnose schizophrenia, the frequency of many symptoms did not differ between the two groups, and in some cases the symptoms were more prevalent in the bipolar mood disorder group. While auditory hallucinations were more frequent in individuals with schizophrenia, those diagnosed with bipolar mood disorder were more likely to demonstrate bizarre social/sexual behavior and symptoms of positive formal thought disorder, such as pressure of speech and derailment. Delusional symptoms did differ between the two groups, but only in type and not in overall frequency. Patients of schizophrenia were more likely to have delusions of reference and persecution, whereas patients of bipolar mood disorder were more likely to have delusions of grandeur and religious delusions. Thus, the positive symptoms of schizophrenia do not seem to be particular to individuals with schizophrenia, but occur quite frequently in bipolar disorder. The negative symptoms of schizophrenia, however, were shown to differentiate the two groups. All five categories of negative symptoms differed between the groups, with schizophrenia patients consistently having a higher prevalence of these symptoms.

When examining the manic and depressive symptoms typically associated with bipolar mood disorder these symptoms were found to differentiate between the two groups, but not in the expected way. Overall, manic symptoms were found to be more prevalent in the bipolar mood disorder groups but depressive symptoms were more frequent in the schizophrenia group. Almost half of the manic symptoms were shown to differ between the groups, but only four of seventeen depressive symptoms differed.

Therefore, it seems that both positive/manic symptoms and negative/depressive symptoms are present in individuals diagnosed with schizophrenia and with bipolar mood disorder and, consequently, they do not present a reliable means of differentiating between these two groups. Negative and depressive symptoms were more prevalent in the groups diagnosed with schizophrenia, whereas manic symptoms and some positive symptoms were more prevalent in the group diagnosed with bipolar mood disorder. These findings seem to suggest, as some have proposed e.g., Hafner et al. that these two disorders are in fact two points on a single continuum of mental illness [9]. This is also consistent with the findings in the present research that almost two thirds of the sample had overlapping symptoms of the two disorders (as indicated by symptom levels above clinical cut-offs). Sixty-five percent of those diagnosed with schizophrenia and $61 \%$ of those diagnosed with bipolar mood disorder had symptoms that overlapped to a clinically significant degree with the other disorder. Additionally, patients in the schizophrenia group were more likely to have overlapping symptoms of both mania and depression than to have overlapping symptoms on only one of these scales.

These findings have many implications for the ways in which mental illness is conceptualized and classified. Treatment efforts and interventions may be enhanced if a more dimensional approach to diagnosing mental illness is utilized. In particular, the recognition is that individuals diagnosed with either schizophrenia or bipolar mood disorder may have both psychotic/manic symptoms and negative/depressive symptoms that need to be addressed. Further theoretical and empirical work is required to determine the most effective way to conceptualize mental illness that best informs treatment.

\section{ACKNOWLEDGEMENTS}

Kristen J. Terpstra, Department of Psychiatry and Behavioral Neurosciences, Mc Master University, 1280 Main St West, Hamilton, Ontario, L8S 4L8.

\section{REFERENCES}

[1] Kraepelin, E., (1919) Dementia praecox and paraphrenia. Chicago Medical Book Co., Chicago.

[2] Kendler, K.S., Karkowski, L.M. and Walsh, D. (1998) The structure of psychosis: Latent class analysis of probands from the Roscommon Family Study. Archives of General Psychiatry, 55, 492-509. http://dx.doi.org/10.1001/archpsyc.55.6.492

[3] Craddock, N., O’Donovan, M.C. and Owen, M.J. (2006) Genes for schizophrenia and bipolar disorder? Implica- 
tions for psychiatric nosology. Schizophrenia Bulletin, 32, 9-16. http://dx.doi.org/10.1093/schbul/sbj033

[4] Ivleva, E., Thaker, G. and Tamminga, C.A. (2008) Comparing genes and phenomenology in the major psychoses: Schizophrenia and bipolar 1 disorder. Schizophrenia Bulletin, 34, 734-742.

http://dx.doi.org/10.1093/schbul/sbn051

[5] Chen, Y.R., Swann, A.C. and Johnson, B.A. (1998) Stability of diagnosis in bipolar disorder. Journal of Nervous \& Mental Disease, 186, 17-23. http://dx.doi.org/10.1097/00005053-199801000-00004

[6] Singh, S.P., Croudace, T., Amin, S., Kwiecinski, R., Medley, I., Jones, P.B. and Harrison, G. (2000) Threeyear outcome of first-episode psychoses in an established community psychiatric service. British Journal of Psychiatry, 176, 210-216. http://dx.doi.org/10.1192/bjp.176.3.210

[7] Cardno, A.G., Rijsdijk, F.V., Sham, P.C., Murray, R.M. and McGuffin, P. (2002) A twin study of genetic relationships between psychotic symptoms. American Journal of Psychiatry, 159, 539-545. http://dx.doi.org/10.1176/appi.ajp.159.4.539

[8] Sim, K., Swapna, V., Mythily, S., Mahendran, R., Kua, E.H., McGorry, P. and Chong, S.A. (2004) Psychiatric co-morbidity in the first episode of psychosis: The Early Psychosis Intervention Program (EPIP) experience. Acta Psychiatrica Scandinavica, 109, 23-29. http://dx.doi.org/10.1111/j.0001-690X.2004.00196.x

[9] Hafner, H., Maurer, K., Trendler, G., an der Heiden, W., Schmidt, M. and Konnecke, R. (2005) Schizophrenia and depression: Challenging the paradigm of two separate diseases-A controlled study of schizophrenia, depression and healthy controls. Schizophrenia Research, 77, 11-24. http://dx.doi.org/10.1016/j.schres.2005.01.004

[10] Romm, K.L., Rossberg, J.I., Berg, A.O., Barrett, E.A., Faerden, A., Agartz, I., Andreassen, O.A. and Melle, I. (2010) Depression and depressive symptoms in first episode psychosis. Journal of Nervous \& Mental Disease,
198, 67-71. http://dx.doi.org/10.1097/NMD.0b013e3181c81fc0

[11] Johnson, J., Horwath, E. and Weissman, M.M. (1991) The validity of major depression with psychotic features based on a community study. Archives of General Psychiatry, 48, 1075-1081.

http://dx.doi.org/10.1001/archpsyc.1991.0181036003900 $\underline{6}$

[12] Schatzberg, A.F. and Rothschild, A.J. (1992) Psychotic (delusional) major depression: Should it be included as a distinct syndrome in DSM-IV? American Journal of Psychiatry, 149, 733-745

[13] Kasanin, J. (1933) The acute schizoaffective psychoses. American Journal of Psychiatry, 90, 97-126.

[14] Heckers, S. (2009) Is schizoaffective disorder a useful diagnosis? Current Psychiatry Reports, 11, 332-337. http://dx.doi.org/10.1007/s11920-009-0048-3

[15] Andreasen, N.C. (1984) The Scale for the assessment of Positive Symptoms (SAPS). The University of Iowa, Iowa City.

[16] Andreasen, N.C. (1983) The Scale for the Assessment of Negative Symptoms (SANS). The University of Iowa, Iowa City.

[17] Hamilton, M. (1960) A rating scale for depression. Journal of Neurology, Neurosurgery \& Psychiatry, 23, 56-62. http://dx.doi.org/10.1136/jnnp.23.1.56

[18] Young, R.C., Biggs, J.T., Ziegler, V.E. and Meyer, D.A. (1978) A rating scale for mania: Reliability, validity and sensitivity. British Journal of Psychiatry, 133, 429-435. http://dx.doi.org/10.1192/bjp.133.5.429

[19] Dratcu, L. and Cali, H.M. (1989) The dexamethasone suppression test: Its relationship to diagnoses, severity of depression and response to treatment. Progress in NeuroPsychopharmacology \& Biological Psychiatry, 13, 99117. http://dx.doi.org/10.1016/0278-5846(89)90007-9 